Vol. 5, No. 1, 2019

Mykola Vorobyov ${ }^{1}$, Denis Prokopenko ${ }^{2}$

1. Department of Applied Mechanics, Ivano-Frankivsk National University of Oil and Gas, 15, Karpatska Str., Ivano-Frankivsk, Ukraine, E-mail: teormech@nung.edu.ua

2. Department of Applied Mechanics, Ivano-Frankivsk National University of Oil and Gas, 15, Karpatska Str., Ivano-Frankivsk, Ukraine, E-mail: Deniss_2005@ukr.net

\title{
ELEMENTS OF THE THEORY OF CLOSED-LOOP MECHANISMS FORMED BY ROLLING BODIES WITHOUT KINEMATIC SLIDING
}

\author{
Received: April 05, 2019 / Revised: June 25, 2019 / Accepted: June 26, 2019
}

(C) Vorobyov M., Prokopenko D., 2019

Abstract. In the modern industry, it is impossible to do without high-tech equipment, for the designing of which the practical experience and experience of scientists are used. The main principles of engineering mechanics are based on the improvement of technical processes that promote creating certain conditions for the development of the manufacturing industry. An innovative approach to solving the problems of designing and creating any equipment makes it possible to combine new technologies and scientific and technical studies.

When designing process equipment, there is a need not only to improve the existing types but also to search for new principles for connecting the supporting elements. It is important to create the right conditions for the effective functioning of reliable mechanisms.

The main elements of the process equipment are the supporting ones. Among them, the bearings are the most important elements. Their reliable and long-term operation depends on the structural features of the supports.

When designing the bearing units, they take into account the purpose of the mechanism, its operating conditions, the magnitude and direction of the loadings applied to the bearings, the required resource, the rotation frequency, the temperature and the state of the environment.

In real bearings, there are no two fixed guides, and the separation is ensured by an intermediate element, which is connected by sliding friction. Besides, modern oil and gas equipment also uses radial supports through which a working fluid circulates with impurities of abrasive particles, which quickly destroys this element. Therefore, there is a need to create a scientific basis for the design of fundamentally new modern bearings.

The purpose of the work is to find the optimum range of existence of closed-loop mechanisms formed by rolling bodies without kinematic sliding and to obtain the analytical dependences that relate the geometric parameters of the bearings to the separation by the rolling bodies in the absence of sliding. In this case, all three drive types are considered: from the outer or inner clip or the two clips together. To ensure the workability of all types of radial bearings, a range of specific basic conditions should be met.

Given the significant advantages of these types of mechanisms, the challenge arises for their further study, with a view to wide implementation and practical application. The results can be useful for designing support elements for equipment used in many manufacturing fields.

Keywords: mechanism, rolling bodies, bearing, kinematic sliding, sliding friction.

\section{Introduction}

One of the most important tasks facing the technical support of the agro-industrial complex in Ukraine is to improve the operational properties of the mechanisms and machines by increasing the reliability, durability, and economy. 


\section{Mykola Vorobyov, Denis Prokopenko}

Reliability and durability are largely due to the phenomena of friction and wear that occur in friction systems. The main ways to traditionally solve friction reduction problems are to use various types of fluids to lubricate the friction units or to select the friction couple materials. But greater results can be achieved by choosing rational design ratios of sizes and links between sections.

\section{Problem Statement}

The operational specificity of the radial bearing with the separation by the rolling bodies requires the solution of the primary task, namely obtaining a rational relationship between its parameters for the most used types of actuation of the mechanism sections.

\section{Review of Modern Information Sources}

Increasing the technical level of roller bearings by the contact endurance of rolling surfaces within a given size is achieved by improving the load distribution between the rollers, optimizing the geometric shape of the rollers, and increasing the number of rollers [1]. Attempts to improve existing bearings by optimizing the face radius of the rollers are described in [2] and [3]. The influence of increasing the number of rollers, as one of the most promising ways of increasing the technical level within a given size, has been investigated for cylindrical bearings in publications [4] and [5].

But Donald Wilkes, an inventor from the United States of America, has patented a new mechanism that has virtually no drag and slip between the band and the rollers, and therefore no friction. It consists of two rollers on opposite sides of the track made of flexible material in the form of a letter $S$. He called his invention Rolamite [6] (Fig. 1).

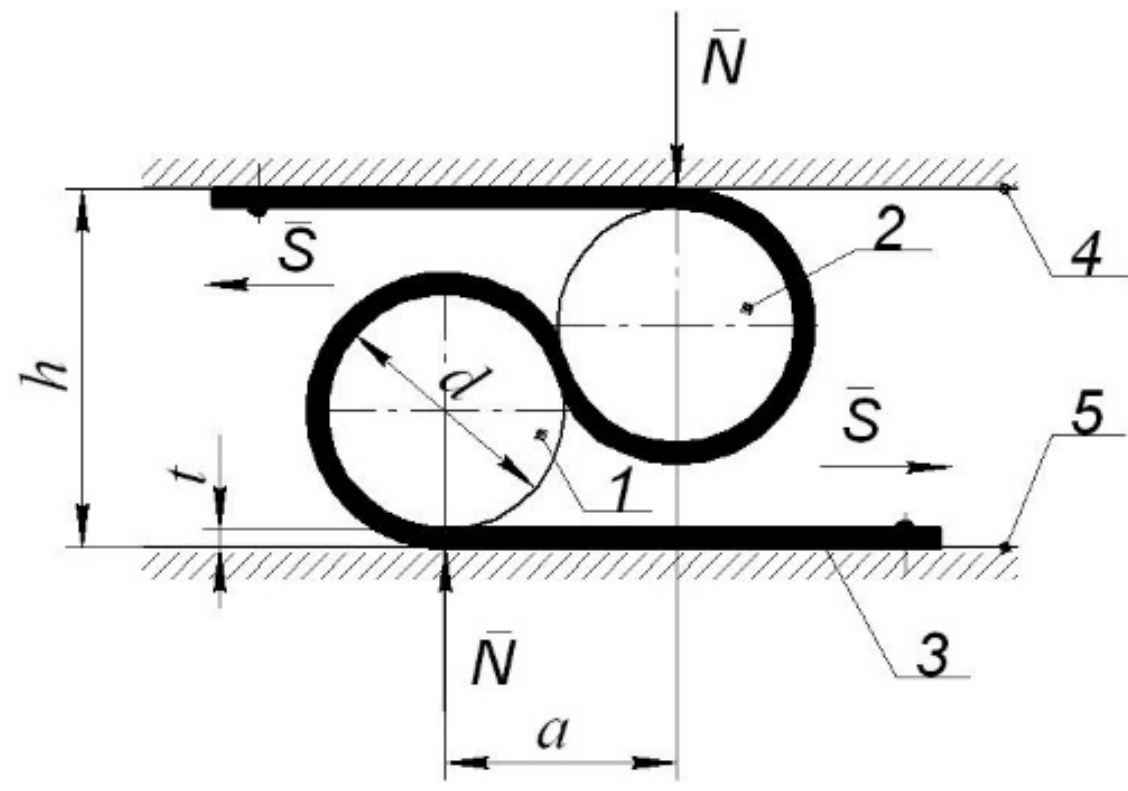

Fig. 1. The classic Rolamite

Here, the rollers 1 and 2 carry out a plane-parallel motion, and the flat guide surfaces 4 (upper) and 5 (lower) are stationary. A band 3 of the thickness $t$ is pre-tensioned by forces $F_{0}$ and its ends are fixed to the surfaces. Such a mechanism can be attributed to an unconventional group of mechanisms with geometrically-force closed-loop contours of elements of kinematic pairs [7].

There are many functions in this device: it is capable of working as a valve, pump, switch, thermostat, coupling, amplifier, brake, pressure sensor, electromagnet, fire alarm, etc. [6]. Special studies have shown that the beryllium and copper bands used in the Rolamite are very strong. Even after a hundred thousand bends, they did not begin to show signs of metal fatigue. It was also found that the sliding friction in the mechanism is converted to rolling: the friction coefficient is less than 0.0005 . This is achieved since the flexible band is pulled out, preventing the sliding rollers of the mechanism. This avoids the use of lubricants to reduce friction [8]. 


\section{Elements of the Theory of Closed-Loop Mechanisms Formed by Rolling Bodies ...}

The fact that friction is almost eliminated due to the application of the Rolamite is also proved in the study [9]. None of the alternative mechanisms is capable of ensuring permanent contact between the roller and the band. With no need for lubricants, the Rolamite can be very useful in the face of sudden changes in temperature or vacuum.

However, limiting the rolling bodies to rollers only and the presence of a band reduces the scope of this type of mechanism.

Researchers' attempt to use double-contour touching of stepped rollers to refuse the use of a band is unsuccessful due to the inability to get rid of kinematic sliding between them. Besides, the probability analysis for combined the roller rotation and roller face sliding, for example, in the rolamite rolling bearings, remained unaddressed.

The question of whether it is possible to create a non-axial (asymmetrical) stripless mechanism with single-contact pairs of rollers provided there is no kinematic sliding friction is not studied. The option of performing a Rolamite mechanism in the presence of non-axiality (asymmetry), for example, an electromagnetic roller pump [10] where changes in the volume of chambers are reached due to non-axiality (asymmetry), or the mechanism of the gas meter [11] was not considered.

\section{Formulation of Research Goals and Objectives}

The goal is to substantiate the workability conditions of all types of radial bearings. The tasks are as follows: to check the possibility of creating a mechanism having a kinematic chain with branches of closed-loop contours formed by rolling bodies without sliding, with guides of a variable radius of curvature; to derive the workability conditions of all types of radial bearings.

\section{Presenting Main Material}

Imagine that in nature there are correlations of parameters such that the supporting rolling bodies in interaction with a fixed guide are separated without a band by the separating rolling bodies also in interaction with another fixed guide so that both guides are located non-axially (asymmetrically) (Fig. 2) and at least one guide is of the curved shape of the closed-loop surface of variable radius $r_{\mathrm{i}}$, for example, for a separating body that would be rolled over a fixed cam spike, that is, the mechanism became a mechanism with a geometric closed-loop contour of elements of kinematic pairs.

Denote the instantaneous axis of rotation of the supporting and separating body by the letter $\Pi_{21}$, the supporting radius $\mathrm{r}_{1}$ with the guide $-P_{13}$ of the radius $R$, the separating radius $r_{2}-P_{2_{\mathrm{B}}}$. The separating body should be directed by a spike.

In the absence of kinematic sliding between the supporting and separating rolling bodies, the point $P_{2 \mathrm{~B}}$ must be located on the line connecting points $\Pi_{21}$ and $P_{13}$.

This is possible if the following ratio is satisfied:

$$
\frac{\Pi_{21} \cdot P_{31}}{\Pi_{21} \cdot P_{2 b}}=\frac{\omega_{1}}{\omega_{2}} .
$$

From the regularities of planetary motion of rolling bodies, we have

$$
\omega_{1}=\left(1-\frac{R}{r_{1}}\right) \cdot \frac{\Omega_{1}}{\Omega_{2}},
$$

and

$$
\omega_{2}=\left(1+\frac{r_{i}}{r_{2}}\right) \cdot \Omega_{2},
$$

where $\Omega_{1}$ and $\Omega_{2}$ are angular velocities of rotation of the centers $O_{1}$ and $O_{2}$ of the rolling bodies.

Substituting (2) and (3) into (1) we obtain after transformations

$$
r_{i}=\left(r_{1}-R\right) \cdot \frac{\Omega_{1}}{\Omega_{2}}-r_{2} .
$$




\section{Mykola Vorobyov, Denis Prokopenko}

To determine $r_{i}$ we use the formula for calculating the curvature of a plane curve at the point $P_{2 \mathrm{i}}$ $\left(x_{p 2 i}, \mathrm{y}_{p 2 i}\right)$

$$
r_{i}=\frac{\left[1+\left(y_{p 2 i}^{\prime}\right)^{2}\right]^{\frac{3}{2}}}{y_{p 2 i}^{\prime \prime}} .
$$

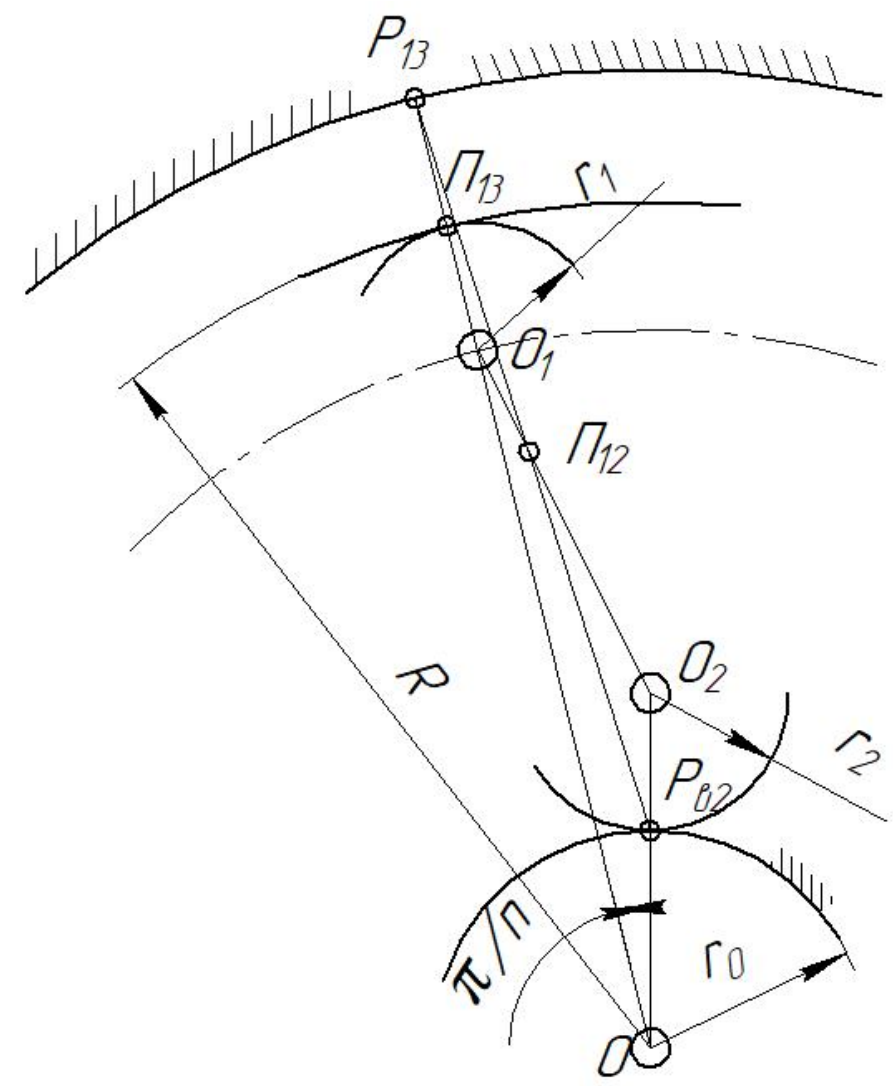

Fig. 2. The calculation scheme

We assume that $y_{p 2 i}^{\prime}=p$, then $y_{p 2 i}^{\prime \prime}=p^{\prime}=\frac{d y_{20}}{d x_{p 2 i}}$ and $\frac{r_{i} d p}{\left(1+p^{2}\right)^{\frac{3}{2}}}=d x_{p 2 i}$, after that

$$
x_{p 2 i}=\frac{r_{i} p}{\sqrt{p^{2}+1}}+C_{1}
$$

or

$$
\left(x_{\Pi 2 i}-C_{1}\right)^{2}\left(p^{2}+1\right)=r_{i}^{2} p^{2},
$$

and

$$
\begin{aligned}
& \left(x_{p 2 i}-C_{1}\right)^{2} p^{2}+\left(x_{p 2 i}-C_{1}\right)^{2}-r_{i}^{2} p^{2}=0, \\
& {\left[r_{i}^{2}-\left(x_{p 2 i}-C_{1}\right)^{2}\right] p^{2}=\left(x_{p 2 i}-C_{1}\right)^{2}=0 .}
\end{aligned}
$$

Then

$$
p=\frac{x_{p 2 i}-C_{1}}{\sqrt{r_{i}^{2}-\left(x_{p 2 i}-C_{1}\right)^{2}}}
$$


Elements of the Theory of Closed-Loop Mechanisms Formed by Rolling Bodies ...

Let us denote $x_{p 2 i}-C_{1}=\mu$, then

and the solution is as follows:

$$
\frac{d y_{02 i}}{d x_{p 2 i}}=\frac{\mu}{\sqrt{r_{i}^{2}-\mu^{2}}}
$$

$$
y_{p 2 i}=\int \frac{\mu d x_{\Pi 20}}{\sqrt{r_{i}^{2}-\mu^{2}}} .
$$

We assume that $\mu=r_{i} \sin t$, then $d \mu=r_{i} d t \cos t$ and

$$
y_{p 2 i}=\int \frac{r_{i} \sin t \cdot r_{i} \cos t d t}{\sqrt{r_{i}^{2}-r_{i}^{2} \cos ^{2} t}}=\int r_{i} \sin t d t=-r_{i} \cos t+C_{2},
$$

where $t=\arcsin \frac{\mu}{r_{i}}$.

The solution of (6) is as follows:

$$
y_{p 2 i}=C_{2}-r_{i} \cos \left(\arcsin \frac{\mu}{r_{i}}\right)=C_{2}-r_{i} \cdot \sqrt{1-\frac{\mu^{2}}{r_{i}^{2}}}=C_{2}-\sqrt{r_{i}-\mu^{2}},
$$

or

$$
y_{p 2 i}=C_{2}-\sqrt{r_{i}^{2}-\left(x_{p 2 i}-\mathrm{C}_{1}\right)^{2}} .
$$

The coordinates of the center $O_{i}$ of the spike curvature are as follows

$$
\xi=x_{p 20}-\frac{p \cdot\left(1+p^{2}\right)}{p^{\prime}} ; \quad \eta=y_{p 20}+\frac{1+p^{2}}{p^{\prime}},
$$

where

$$
\begin{gathered}
p=\frac{x_{p 20}-C_{1}}{\sqrt{r_{i}^{2}-\left(x_{p 20}-C_{1}\right)^{2}}} ; \\
p^{\prime}=\frac{d^{2} y_{p 20}}{d x_{p 20}^{2}}=\frac{1}{\sqrt{r_{i}^{2}-\left(x_{p 20}-C_{1}\right)^{2}}}+\frac{x_{p 20}-C_{1}}{\left[\sqrt{r_{i}^{2}-\left(x_{p 20}-C_{1}\right)^{2}}\right]^{3}} \cdot\left[-\frac{1}{2} \cdot \frac{-2 \cdot\left(x_{p 20}-C_{1}\right)}{\left[\sqrt{r_{i}^{2}-\left(x_{p 20}-C_{1}\right)^{2}}\right]^{3}}=\frac{r_{i}^{2}}{\left[\sqrt{r_{i}^{2}-\left(x_{p 20}-C_{1}\right)^{2}}\right]^{3}} .\right. \\
=
\end{gathered}
$$

Then

$$
\xi=x_{p 20}-\frac{x_{p 20}-C_{1}}{\sqrt{r_{i}^{2}-\left(x_{p 20}-C_{1}\right)^{2}}} \cdot\left(1+\frac{\left(x_{p 20}-C_{1}\right)^{2}}{r_{i}^{2}-\left(x_{p 20}-C_{1}\right)^{2}}\right) \cdot \frac{\left[\sqrt{r_{i}^{2}-\left(x_{p 20}-C_{1}\right)^{2}}\right]^{3}}{r_{i}^{2}} .
$$

After the transformations we get

$$
\xi=C_{1}=\text { const }
$$




$$
\eta=C_{2}-\sqrt{r_{i}^{2}-\left(x_{p 2 i}-\mathrm{C}_{1}\right)^{2}}+\frac{r_{i}^{2}}{r_{i}^{2}-\left(x_{p 2 i}-\mathrm{C}_{1}\right)^{2}} \cdot \frac{\left[\sqrt{r_{i}^{2}-\left(x_{p 20}-C_{1}\right)^{2}}\right]^{3}}{r_{i}^{2}},
$$

that is

$$
\eta=C_{2}=\text { const }
$$

Equations for $\xi$ and $\eta$ are characteristic of a circle whose equation is

$$
\begin{aligned}
&\left(y_{P 20}-C_{2}\right)^{2}= r_{i}^{2}-\left(x_{P 20}-C_{1}\right)^{2}, \\
& \text { or } \\
&\left(y_{P 20}-C_{2}\right)^{2}+\left(x_{P 20}-C_{1}\right)^{2}=r_{i}^{2} .
\end{aligned}
$$

The center of this circle is located at the point with coordinates $x_{P 20}=C_{1}, y_{P 20}=C_{2}$.

It can be seen that it is not possible to create a mechanism with a closed-loop kinematic chain formed by rolling bodies without sliding, with guides of a variable radius of curvature, and the spike should be oriented axially.

With the central location of the curvature center of the guides, it is possible to create a rolling bearing with the formation of closed-loop energy contours [12] due to the separation by the rolling bodies with the condition that there is no kinematic sliding both in the rotation plane and in the perpendicular direction.

To obtain the analytical dependences that relate the geometric parameters of the bearings to the separation by the rolling bodies in the absence of sliding, let us consider the following design types: the rollers are the supporting rolling bodies, and the balls are the separating ones; the balls are the supporting rolling bodies, and the rollers are the separating ones.

In this case, we consider all three drive types: from the outer or inner clip or the two clips together. To ensure the workability of all types of radial bearings, a range of such conditions should be met.

\section{The condition of absence of contact of bodies with each other}

The operational specificity of the radial bearing with the separation by the rolling bodies requires the solution of the primary task, namely obtaining a rational relationship between its parameters for the most used types of actuation of the mechanism sections.

These include ball-roller and roller-ball types with movable inner, outer, or both rings, in which rollers would not rub their faces to the rings. In this case, their workability should be ensured.

The following uniform conditions should be met for all types of radial bearings.

Conditions of the absence of contact of supporting bodies with each other:

$$
\begin{aligned}
\left(y_{P 20}-C_{2}\right)^{2}= & r_{i}^{2}-\left(x_{P 20}-C_{1}\right)^{2}, \\
& \text { or } \\
\left(y_{P 20}-C_{2}\right)^{2}+ & \left(x_{P 20}-C_{1}\right)^{2}=r_{i}^{2},
\end{aligned}
$$

where $\delta$ is the face gap between adjacent supporting bodies (Figs. 2 and 3 ).

The condition of placement of the separating rolling body in the gap between the supporting bodies without slipping:

$$
r_{1}>r_{2}>\delta
$$

The condition of avoidance of contact of each supporting rolling body with both outer and inner rings:

$$
r_{1}<R-r_{0} .
$$

The condition of absence of sliding of the rolling bodies depends on which rings (clamps) operate as drives: external and internal at the same time, only external or internal only or the drive moves the center of rotation of the rolling bodies. 


\section{The condition of absence of kinematic sliding on the forming surfaces of rolling bodies driven by outer and inner rings}

This kinematic connection belongs to differential mechanisms. In it, the relative rotation pole $\Pi_{13}$ of the sections 3 and 1 (Fig. 3) should be located on the line connecting the instantaneous centers of velocities $O$ and $P_{10}$, and the relative pole $\Pi_{2 b}$ of the sections 2 and b - on the line $O P_{20}$.

Since the positions of the instantaneous velocity center $O$ and the relative pole $\Pi_{13}$ are known, then the instantaneous velocity center $P_{10}$ should be positioned such that the relative pole $\Pi_{13}$ would divide the distance $O P_{10}$ by segments inversely proportional to the absolute angular velocities $\omega_{3}$ of the outer ring of the supporting rolling body $1: \frac{\omega_{3}}{\omega_{1}}=\frac{\Pi_{31} P_{10}}{R}$, after that $\omega_{1}=\frac{R \omega_{3}}{\Pi_{31} P_{10}}$, where $\Pi_{31} P_{10}=r_{1}-O_{1} P_{10}$. In the pair of sections 2 and $\mathrm{b}$, the relative pole $\Pi_{2 b}$ should divide the distance $O P_{20}$ by internally: $\frac{\omega_{2}}{\omega_{b}}=\frac{r_{0}}{O_{2} P_{20}+r_{2}}$, after that $\omega_{2}=\frac{\omega_{b} r_{0}}{O_{2} P_{20}+r_{2}}$.

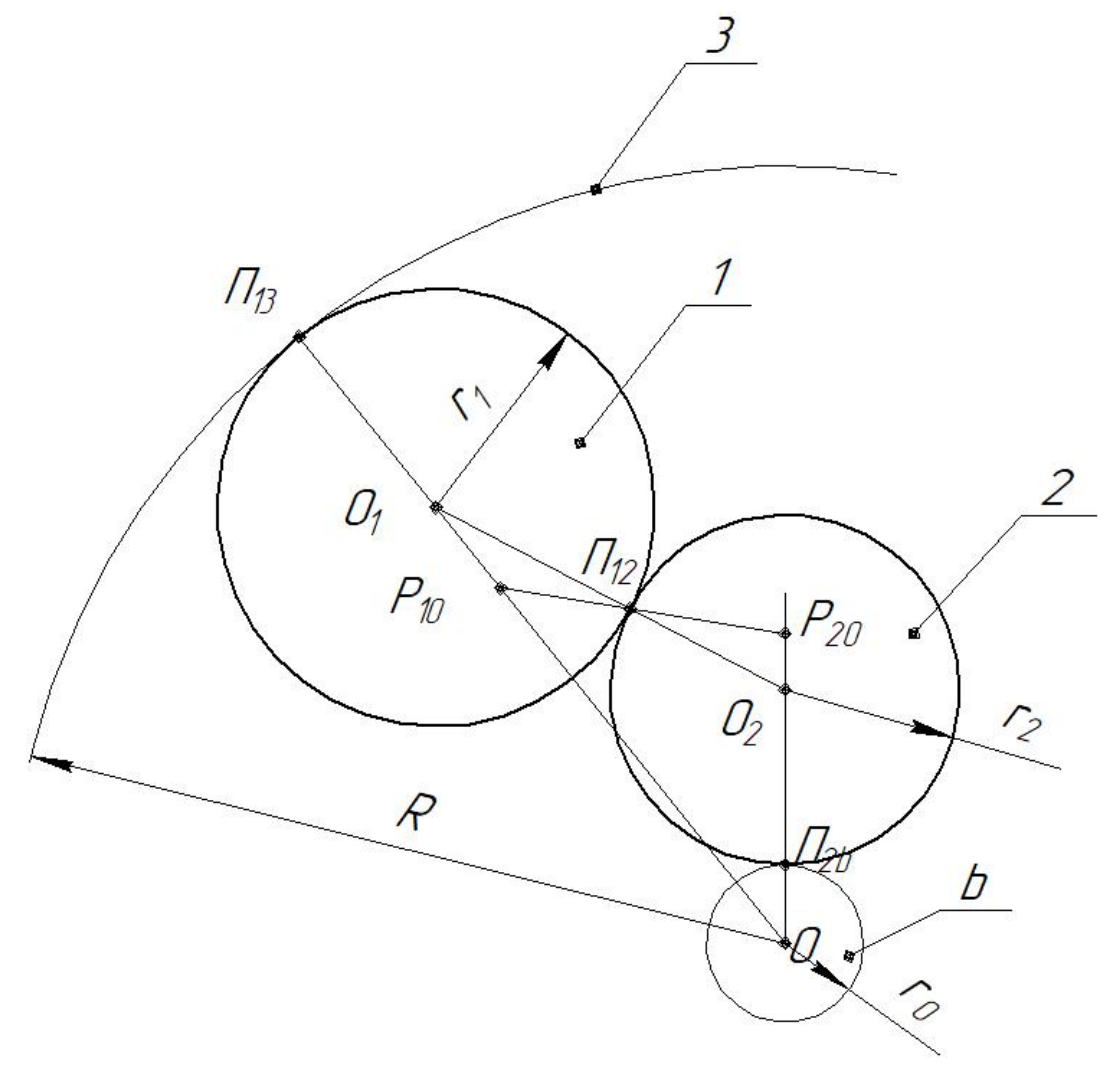

Fig. 3. Kinematic connection with the two-ring drive

Absolute angular velocities $\omega_{1}$ and $\omega_{2}$ can also be found from the equations of inversion of the sections motion by converting the differential mechanism into the planetary one, for example, due to a stop of the section $b: \omega_{1}=\left(1-\frac{r_{0}}{r_{1}}\right)\left(\omega_{3}-\omega_{b}\right), \omega_{2}=\left(1+\frac{r_{0}}{r_{1}}\right)\left(\omega_{3}-\omega_{b}\right)$.

Then

$$
O_{1} P_{10}=r_{1} \frac{R}{\left(r_{0}-r_{1}\right)\left(1-k_{b 3}\right)}, O_{2} P_{20}=\frac{k_{b 3} r_{0} r_{1}}{\left(r_{2}+r_{0}\right)\left(1-k_{b 3}\right)}-r_{2},
$$

where $k_{b 3}=\frac{\omega_{b}}{\omega_{3}}$.

In order not to slip between the rolling bodies, the condition should be fulfilled 


$$
\Pi_{12} P_{20}=\frac{R\left(r_{0}-r_{1}\right)}{\left(r_{0}+r_{2}\right)\left[\left(r_{0}-r_{1}\right)\left(1-k_{b 3}\right)+R\right]} \Pi_{12} P_{10} .
$$

The sections in the mechanism should move so that the angle is equal $\gamma=2 \pi / n$, where $n$ is the total number of rolling bodies. Then by the cosine theorem we obtain

$$
\left(\Pi_{12} P_{10}+\Pi_{12} P_{20}\right)^{2}=\left(R-r_{1}-O_{1} P_{10}\right)^{2}+\left(r_{0}+r_{2}+O_{2} P_{20}\right)^{2}-2\left(R-r_{1}-O_{1} P_{10}\right)\left(r_{0}+r_{2}+O_{1} P_{10}\right) \cos \gamma
$$

For the cosine of the angle at $\Pi_{12}$ we can write the ratio

$$
\left(\Pi_{12} P_{20}^{2}+r_{2}^{2}-P_{20} O_{2}^{2}\right) r_{2}^{-1} \Pi_{12} P_{20}{ }^{-1}=\left(\Pi_{12} P_{10}{ }^{2}+r_{1}^{2}-P_{10} O_{1}^{2}\right) r_{1}^{-1} \Pi_{12} P_{10}{ }^{-1} .
$$

Substituting the values of all the segments into the last two formulae, we finally get

$$
\begin{gathered}
\frac{r_{1}^{2}\left\{k_{b 3} r_{1} r_{0}-r_{2}\left(r_{0}+r_{2}\right)\left(1-k_{b 3}\right)\left(r_{0}-r_{1}\right)+r_{2} R\left(r_{0}+r_{2}\right)\left[\left(r_{1}-r_{0}\right)\left(1-k_{b 3}\right)-R\right]\right\}}{R\left(r_{0}-r_{1}\right)\left\{R\left(r_{0}-r_{1}\right) r_{1}-r_{2}\left(r_{0}+r_{2}\right)\left[r_{1}\left(r_{0}-r_{1}\right)\left(1-k_{b 3}\right)+R\right]\right\}}= \\
=\left(r_{0}+r_{2}\right)^{2}\left\{\frac{\left[\left(R-r_{1}\right)\left(r_{0}-r_{1}\right)\left(1-k_{b 3}\right)-r_{1} R\right]^{2}+\left[\left(r_{0}+r_{2}\right)^{2}+k_{b 3} r_{0} r_{1}\right]^{2}}{\left(r_{0}-r_{1}\right)\left\{\left(r_{0}+r_{2}\right)\left[\left(r_{0}-r_{1}\right)\left(1-k_{b 3}\right)-R\right]+R\left(r_{0}-r_{1}\right)\right\}}-\right. \\
\left.-\frac{2\left[\left(R-r_{1}\right)\left(r_{0}-r_{1}\right)\left(1-k_{b 3}\right)-R r_{1}\right]\left[\left(r_{0}+r_{2}\right)^{2}+k_{b 3} r_{0} r_{1}\right] \cos 2 \pi / n_{0}}{\left(r_{0}-r_{1}\right)\left\{\left(r_{0}+r_{2}\right)\left[\left(r_{0}-r_{1}\right)\left(1-k_{b 3}\right)-R\right]+R\left(r_{0}-r_{1}\right)\right\}}\right\}
\end{gathered}
$$

where $n=\frac{2 \pi}{\arccos \left[\frac{2 r_{0}\left(R-r_{0}\right)}{\left(R-r_{1}\right)^{2}-\left(r_{0}+r_{2}\right)^{2}+r_{0}^{2}}\right]}$ is the number of supporting rolling bodies equal to the number of separating rolling bodies.

The obtained formula allows you to choose the ratio of the parameters $r_{0}, r_{1}, r_{2}, R$, and $n$ at the condition of ensuring the absence of the sliding on forming surfaces of the rolling bodies with each other, as well as on the surfaces of the rings (clips). As you can see, this ratio depends on the ratio of the input velocities. Their changes should be made so that $k_{b 3}$ does not change. The number of supporting rolling bodies $n$ for all drive variants is determined equally.

\section{The condition of absence of kinematic sliding on the forming surfaces of the rolling bodies driven by the outer ring}

In this case, the mechanism will be planetary. We stop section 3 (the outer ring) because it drives all other sections. Then the instantaneous velocity center $P_{20}$ will start to rotate in the opposite direction about $\mathrm{O}_{2}$. The absolute instantaneous velocity center $P_{10}$ (Fig. 4) for this mechanism should be located not on line $P_{20} \Pi_{13}$ but above the relative pole $\Pi_{13}$.

It will divide the distance $P_{20} P_{10}$ internally into segments inversely proportional to the absolute angular velocities of the outer ring and the supporting rolling body 1 .

Applying the Willis formula, we obtain the relation: $i_{20}^{(3)}=\frac{\omega_{2}-\omega_{3}}{-\omega_{3}}=-\frac{r_{0}}{r_{2}}, i_{21}^{(3)}=\frac{\omega_{2}-\omega_{3}}{\omega_{1}-\omega_{3}}=-\frac{r_{1}}{r_{2}}$, after that $\omega_{2}=\frac{r_{2}+r_{0}}{r_{2}} \omega_{3} ; \omega_{1}=\frac{r_{1}-r_{0}}{r_{1}} \omega_{3} ; P_{10} \Pi_{13}=\frac{R r_{1}}{r_{1}-r_{0}}$, because $\frac{\omega_{3}}{\omega_{1}}=\frac{\Pi_{31} P_{10}}{R}$.

To avoid this sliding, the condition of coincidence of the point of intersection of the lines $P_{10} P_{20}$ and $O_{1} O_{2}$ with the point $\Pi_{12}$ should be fulfilled, where the velocities of the contacting rolling bodies will be the same: $\omega_{1} \Pi_{12} P_{10}=\omega_{2} \Pi_{12} P_{20}$, after that $\Pi_{12} P_{20}=\Pi_{12} P_{10} \omega_{1} / \omega_{2}=\Pi_{12} P_{10} \frac{\left(r_{1}-r_{0}\right) r_{2}}{\left(r_{2}+r_{0}\right) r_{1}}$.

We use the angle maintaining requirement $\gamma=2 \pi / n$ : 
Elements of the Theory of Closed-Loop Mechanisms Formed by Rolling Bodies ...

$$
\left(\Pi_{12} P_{20}+\Pi_{12} P_{10}\right)^{2}=\left(R+\Pi_{13} P_{10}\right)^{2}+r_{0}^{2}-2\left(R+\Pi_{31} P_{10}\right) r_{0} \cos (2 \pi / n) .
$$

From the expression for the cosine of the angle at $\Pi_{12}$ we get $\Pi_{12} P_{10}{ }^{2}=\Pi_{13} P_{10} \frac{\left(r_{2}+r_{0}\right)\left(2 r_{1}+\Pi_{13} P_{10}\right)}{r_{2}+2 r_{0}-r_{1}} \quad$ or after substitution of values $\Pi_{12} P_{10}$ and $\Pi_{13} P_{10}$, and after simple transformations, we finally get

$$
\begin{aligned}
& R\left[2\left(r_{1}-r_{0}\right)+R\right]\left[r_{1}^{2}\left(r_{2}+r_{0}\right)^{2}+r_{2}^{2}\left(r_{1}-r_{0}\right)^{2}\right]=\left(r_{2}+r_{0}\right)\left(r_{2}+2 r_{0}-\right. \\
& \left.-r_{1}\right)\left[R^{2}\left(2 r_{1}-r_{0}\right)^{2}+r_{0}^{2}\left(r_{1}-r_{0}\right)^{2}-2 R\left(r_{1}-r_{0}\right)\left(2 r_{1}-r_{0}\right) r_{0} \cos 2 \pi / n\right] .
\end{aligned}
$$

This formula allows in the design stage to determine the ratio of the parameters $r_{0}, r_{1}, r_{2}, R$, and $n$ at the condition of ensuring the absence of sliding on forming surfaces of the rolling bodies with each other, as well as on the surfaces of the rings (clips). For this kind of bearing design, the rolling bodies should be stepped.

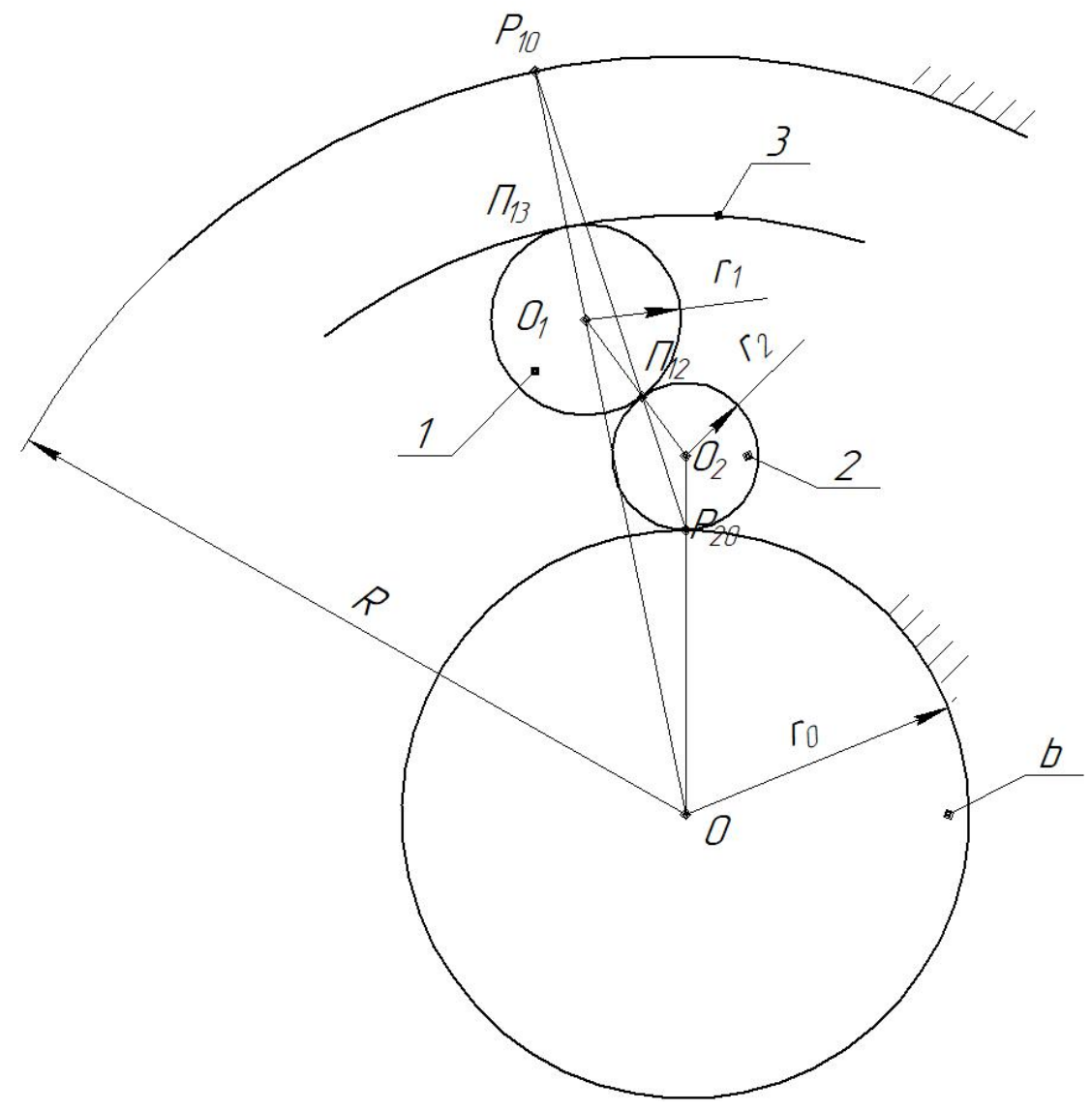

Fig. 4. Kinematic connection to the outer ring drive

The condition of absence of kinematic sliding on the forming surfaces of the rolling bodies driven by the inner ring

In this case, the mechanism will also be planetary. If we move all the sections with a velocity $-\omega_{b}$ (Fig. 5), then from the formulae: $\frac{\omega_{1}-\omega_{b}}{-\omega_{b u}}=\frac{R}{r_{1}}, \frac{\omega_{1}-\omega_{b}}{\omega_{2}-\omega_{b}}=-\frac{r_{2}}{r_{1}} \quad$ we get $\quad \omega_{2}=\frac{r_{2}+R}{r_{2}} \omega_{b}$; $\omega_{1}=r_{2}-\frac{r_{2} r_{0}}{r_{2}+R} \omega_{3} ; O_{2} P_{20}=r_{2}-\frac{r_{0} r_{2}}{r_{2}+R}$.

To avoid the sliding of the rolling bodies, the condition of coincidence of the point of intersection of the lines $P_{10} P_{20}$ and $O_{1} O_{2}$ with the point $\Pi_{12}$ should be fulfilled, where the velocities of the contacting rolling bodies will be the same: 
$\omega_{1} \Pi_{12} P_{10}=\omega_{2} \Pi_{12} P_{20}$, after that $\Pi_{12} P_{20}=\Pi_{12} P_{10} \omega_{1} / \omega_{2}=\Pi_{12} P_{10} \frac{\left(r_{1}-R\right) r_{2}}{\left(r_{2}+R\right) r_{1}}$.

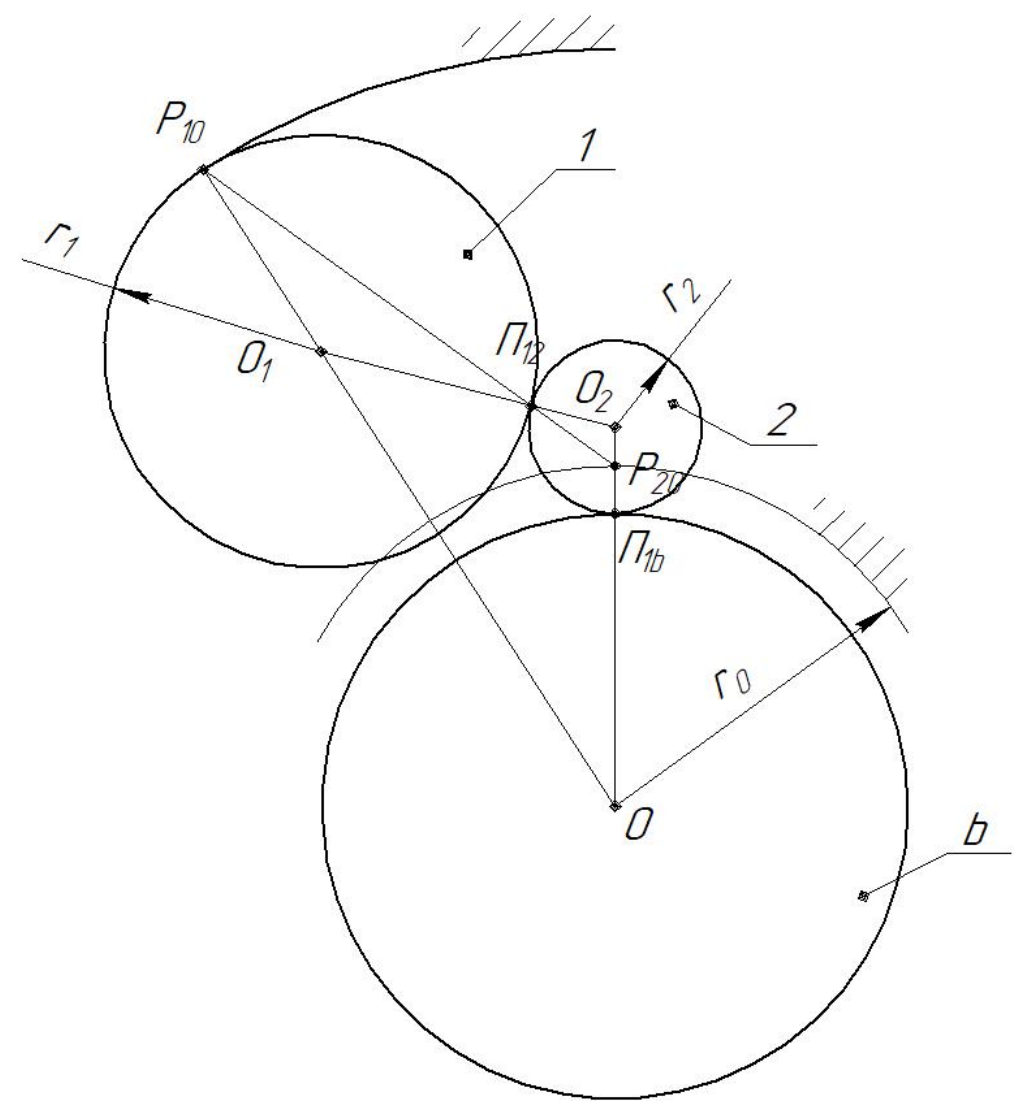

Fig. 5. Kinematic connection to the inner ring drive

Using the angle maintaining requirement $\gamma=2 \pi / n$ we get

$$
\left(\Pi_{12} P_{20}+\Pi_{12} P_{10}\right)^{2}=R^{2}+\left(r_{0}+\frac{r_{0} r_{2}}{r_{2}+R}\right)^{2}-2 R\left(r_{0}+\frac{r_{0} r_{2}}{r_{2}+R}\right) \cos (2 \pi / n) .
$$

After using equality of the cosine of the angle at $\Pi_{12}$ and the value $\Pi_{12} P_{20}{ }^{2}$ we get:

$$
\begin{gathered}
\frac{r_{2}}{r_{1}} \Pi_{12} P_{20} \times \Pi_{12} P_{10}=\Pi_{12} P_{20}^{2}+r_{2}^{2}-O_{2} P_{20}^{2} ; \\
\left(r_{1}-R\right)^{2}\left[R^{2}\left(r_{2}+R\right)^{2}+r_{0}^{2}\left(2 r_{2}+R\right)^{2}-2 R r_{0}\left(r_{2}+R\right)\left(2 r_{2}+R\right) \cos \left(2 \pi / n^{2}\right)\right]\left[\left(r_{2}+\right.\right. \\
\left.+R) r_{1}-\left(r_{1}-R\right) r_{2}\right]\left(r_{2}+R\right)^{2}=r_{1} r_{0}\left(r_{1}-R\right)\left[r_{0}-2\left(r_{2}+R\right)\right]\left[r_{1}^{2}\left(r_{2}+R\right)+r_{2}^{2}\left(r_{1}-R\right)\right] .
\end{gathered}
$$

\section{The condition of absence of kinematic sliding on the forming surfaces of rolling bodies with an axial} drive

Imagine that the drive moves the center of the supporting roller 1 with an angular velocity $\Omega$, and the rollers with the following angular velocities:

$$
\omega_{1}=\frac{R-r_{1}}{r_{1}} \Omega ; \quad \omega_{2}=\frac{r_{2}+r_{0}}{r_{2}} \Omega
$$

Applying the principle of rotation with angular velocity $\Omega$, we obtain $\frac{\omega_{1}-\Omega}{\omega_{2}-\Omega}=-\frac{r_{2}}{r_{1}}$, after that $\omega_{2}=\left[1-\frac{1}{r_{21}}\left(R-2 r_{1}\right)\right] \Omega$

By equating $\omega_{2}$, we will finally get 


\section{Elements of the Theory of Closed-Loop Mechanisms Formed by Rolling Bodies ...}

$$
r_{0}+2 r_{2}=R
$$

which theoretically allows you to find the right ratio between the corresponding radii to avoid kinematic sliding on the cylindrical surfaces.

But the following parameter values give rise to a paradox: the separating and supporting rollers should touch internally the clip of the radius $R$, and the straight lines corresponding to the segments $\Pi_{12} P_{10}$ and $\Pi_{12} P_{20}$ should be parallel. It is known [13] that the mentioned straight lines will be parallel in the classic design of the rolamite-type mechanism, in which the constancy of the contact of the sections is ensured by the $S$-like wrapping of two rollers in the gap between two parallel guides of the frame. Both of these guides are two half-planes facing each other.

The condition of absence of sliding on surfaces of rolling solids will be satisfied for any ratio of the radii $r_{1}$ and $r_{2}$ if the distance $h$ between the half-planes is within $r_{2} \leq h / 2 \leq r_{1}+r_{2}$, if $r_{2}>r_{1}$. But it is impossible to close the contour between $\mathrm{n}$ rolling bodies. Therefore, a pair of rollers requires a band with such pre-tension that would ensure contact of the upper roller with a corresponding half-plane through the band.

\section{Conclusions}

The theory developed is a scientific substantiation for obtaining both mechanisms of a given type and bearings. It was found that:

- it is impossible to create a non-axial (an asymmetrical) mechanism without a band with a geometrically closed contour of the rolling bodies without kinematic sliding between them;

- the use of two-contact rolling due to the touching between adjacent rolling bodies results in kinematic sliding friction, in at least one of the contacts;

- creation on the principle of separation by the rolling bodies of the bearing without a band between them in the absence of sliding in any movable clip requires one or two-stage rollers or dropping of one of the balls into the prismatic groove;

- to guarantee the absence of sliding friction of the roller faces to the clip, you need to use a ball and the shaped part of a roller as an element of a kinematic pair with a ball;

- the maximum reduction of kinematic sliding in a non-axial (an asymmetrical) mechanism can be achieved by choosing rollers as the rolling bodies, the contacts of which in pairs will be similarly separated by an infinite thin elastic band, provided it does not touch the guides in the plane perpendicular to the plane of motion;

- the fixed clips for each of the contacting rolling bodies should be located opposite their contact area;

- the separation of the rolling bodies by the rolling bodies is generally advisable to use in bearings with one drive ring since the simultaneous drive of the outer and inner rings limits the use of the specified separation by certain ratios between their angular velocities.

\section{References}

[1] A. V. Haidamaka, "Udoskonalennia konstruktcii separatoriv dlia pidshypnykiv pidvyshchenoho tekhnichnoho rivnia" ["Improvement of design of separators for bearings of high technical level"], Vostochnojevropejskij zhurnal peredovykh technologij [Eastern-European Journal of Enterprise Technologies], vol.1, no. 6 (84), pp. 53-62, 2016. [in Ukrainian].

[2] E. Simson, V. Ovcharenko, and M. Trokhman, "Modelirovanije i optimizacija iznosa v zone tortcevoho kontakta skol'zhenija rolikovykh podshipnikov" ["Modeling and optimization of wear in the face contact zone of roller bearings during sliding"], MicroCA: Sektcija - Matematychne modeliuvannia v mekhanitci i systemakh [MicroCA: Section - Mathematical modeling in mechanics and systems], vol. 2, pp. 35-36, 2009. [in Russian].

[3] H. Fujiwara, T. Tsujimoto, and K. Yamauchi, "Optimized radius of roller large end face in tapered roller bearings", NTN Technical review, vol. 77, pp. 96-104, 2009.

[4] T. Ozu, "High load capacity cylindrical roller bearings", NTN Technical review, vol. 74, pp. 90-95, 2006. 


\section{Mykola Vorobyov, Denis Prokopenko}

[5] T. Ueno, and T. Matsushita, "Extremely high load capacity tapered roller bearings", NTN Technical review, vol. 77, pp. 73-80, 2009.

[6] N. Carlisle, "The Amazing Rolamite - It Opens the Door for 1000 Inventions," Popular Mechanics, vol. 129, no. 2, pp. 92-95, February 1968.

[7] M. S. Vorobyov, and D. P. Prokopenko, Suchasne pidhruntia vdoskonalennia teorii mekhanismiv [The modern basis for improving the theory of mechanisms]. Ivano-Frankivsk, Ukraine: IFNTUNG Publ., 2019. [in Ukrainian].

[8] D. F. Wilkes, “Roller-band devices”, U.S. Patent 3452 175, June 24, 1969.

[9] "Rolomite reduces friction in devices," Tribology, vol. 1, issue 2, pp. 71-73, March 1968.

[10] M. S. Vorobyov, and D. P. Prokopenko, "Elektromahnitnyi hidronasos kotchennia" [“A hydraulic roller pump with electromagnetic actuator"], UA Patent 108050, March 10, 2015. [in Ukrainian].

[11] M. S. Vorobyov, V. I. Vekeryk, and O. A. Vorobyov, "Rolykovyi litchyl'nyk" ["Roller counter"], UA Patent 68800A, August 16, 2004. [in Ukrainian].

[12] M. S. Vorobyov, Perspektyvni napriamky vdoskonalennia teorii mashyn [Promising directions for improving the theory of machines]. Ivano-Frankivsk: IFNTUNG Publ., 2015. [in Ukrainian].

[13] K. Ragulskis, et. al., Mekhanismy tipa rolamajt [Rolamite-type mechanisms]. Vilnius, Lithuania: Mokslas Publ., 1990. [in Russian]. 